\title{
Inflammasome modulation by Cryptococcus neoformans extracellular vesicles produced in different conditions
}

Clara Luna Freitas Marina 1; Pedro Henrique Miranda Bürgel 1; Daniel Agustinho 2; Daniel Zamith-Miranda 2; Aldo Tavares 1, Joshua Nosanchuk 2; Anamelia Lorenzetti Bocca 1;

1- Applied Immunity Laboratory, Institute of Biology, University of Brasília, Brasília, Distrito Federal, Brazil;

2- Department of Medicine, Albert Einstein College of Medicine, New York, NY, USA

\section{Introduction:}

Cryptococcus neoformans $(\mathrm{Cn})$ is a human pathogenic fungus that infects mainly immunocompromised individuals causing cryptococcosis. Among others, one of its strategies of virulence is the secretion of extracellular vesicles (EVs). It's known that different serotype of $C$. neoformans produce different inflammatory responses when incubated with macrophages and, depending on the growth condition, these fungi differentially release metabolites and virulence factors. In our current project, we show that the culture medium composition significantly impacts the production and biological activity of $C$. neoformans EVs.

\section{Methods}

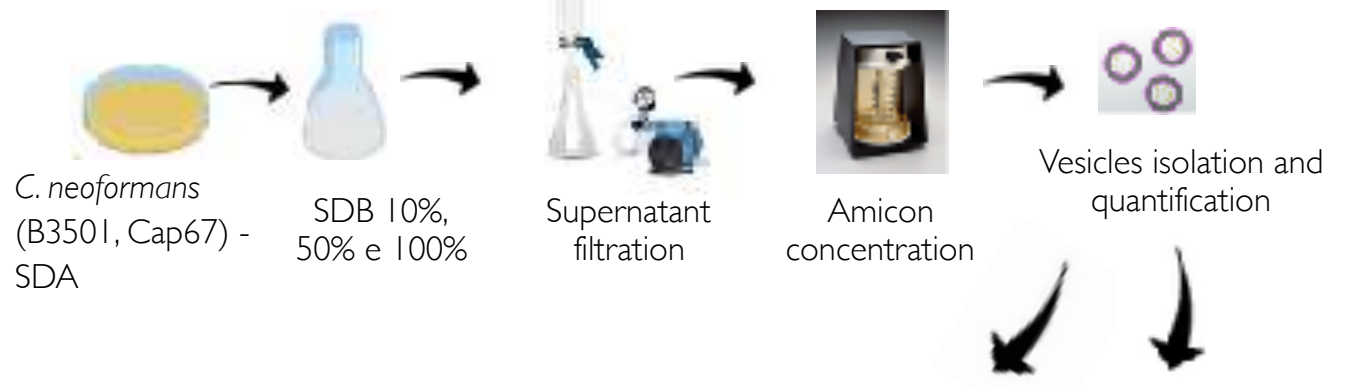

Inflammasome modulation -> ELISA,

RT2 PCR array

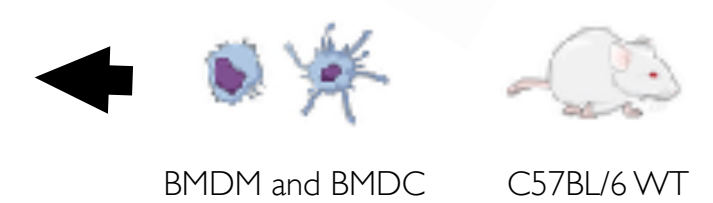

\section{Results}

EVs characterization

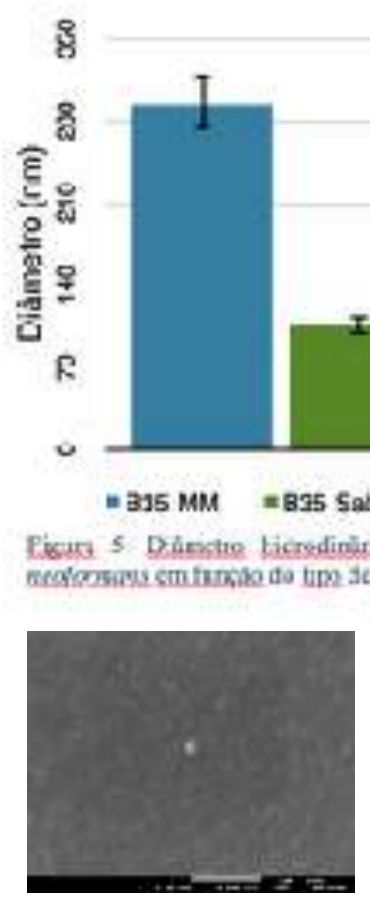

(a) Figure 2. Scanning electron microscopy of the EVs. Cap 67 EVs obtained from SDB $10 \%$ (a),
$50 \%$ (b) and $100 \%$ (c). B3501 EVs obtained from SDB $50 \%$ (d)

\section{Conclusion}

Therefore, we can conclude that the culture medium composition significantly modulates the characteristics of the extracellular vesicles produced by the yeasts. The fact that they are higher when obtained from a poor medium corroborate the hypothesis that these vesicles are virulence factors and are different under nutritional stress situations. Furthermore, the treatment of the infected mice with $\mathrm{Cn}$ vesicles caused a down regulation on several inflammasome genes including IL-1B, TNF- $\boldsymbol{\alpha}$ and IL-18. Hence, these findings are consistent with the loading and release of EVs playing an important role in the fungal stress response, and further link EVs to pathogenesis.
EVs influence on the activation of Inflammasome: (a)

(b)

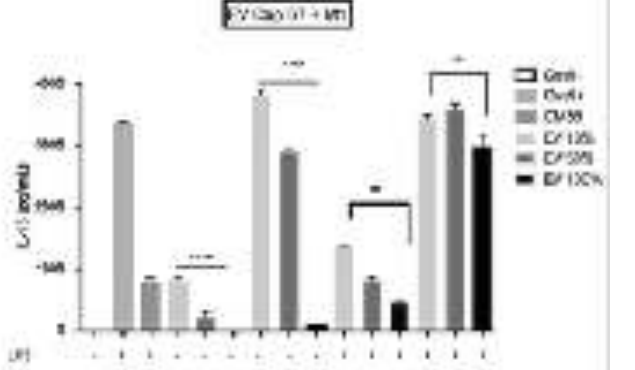

(c)

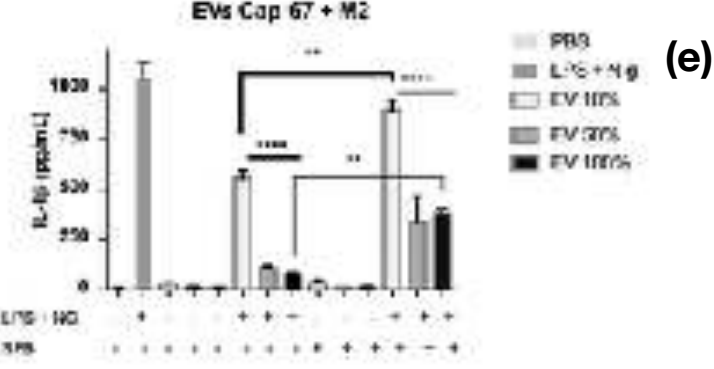

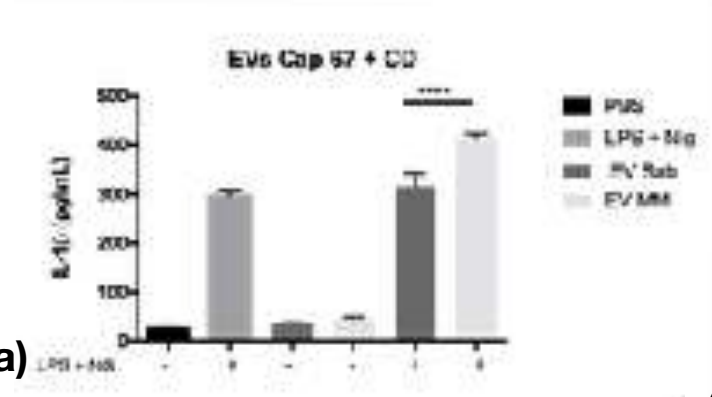

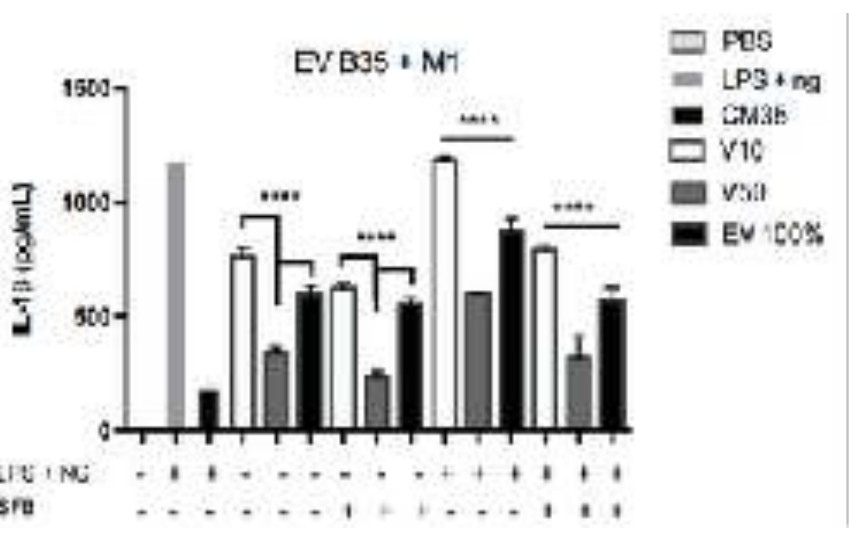

EVB35 $+M 2$

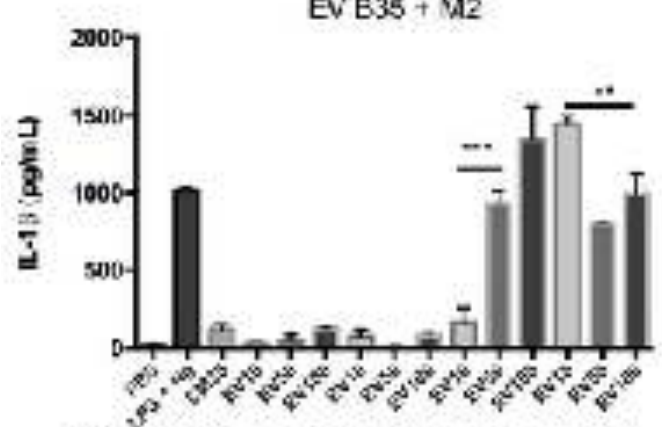

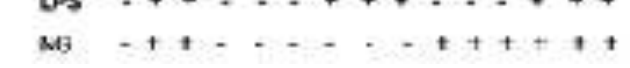

Figure 3. IL-1 $\beta$ production by dendritic cells (a) M1 (b and $d$ ) and M2 macrophages (c and $e$ ) after neoformans Cap 67 (a-c) and B3501 (d and e) growing on Minimum medium (a) and Sabouraud medium at concentrations of $10 \%, 50 \%$ and $100 \%$.

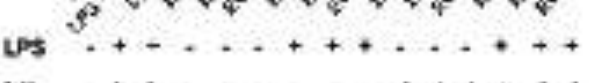

In vivo experiment: infected mice treated with EVs (a)

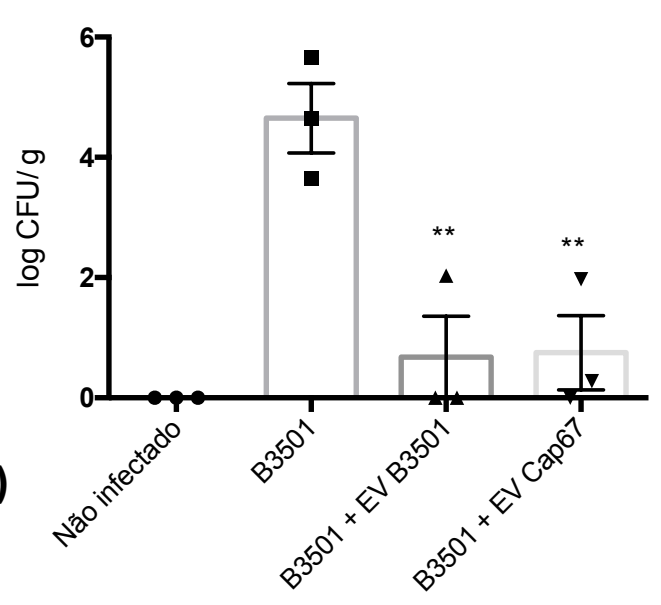

Figure 4. Lungs of infected C57BL/6 mice treated with EVs obtained from B3501 and Cap67 C. neoformans growing on $100 \%$ SDB medium. (a) Lungs CFU. (b) and (c) IL-1 $\beta$ levels in supernatant of the infected lungs measured by ELISA. $\left(^{*}\right)$ means statistically different with $p<0,05$. vesicles of $C$. neoformans varying according to the culture medium

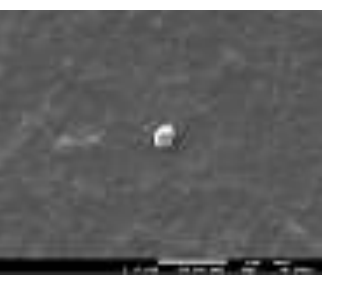

(d)

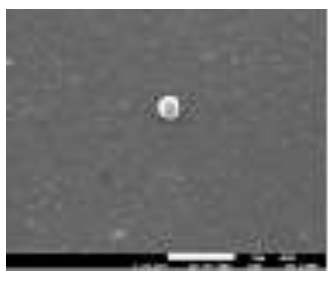

Treated Group Control Group Cn PBS

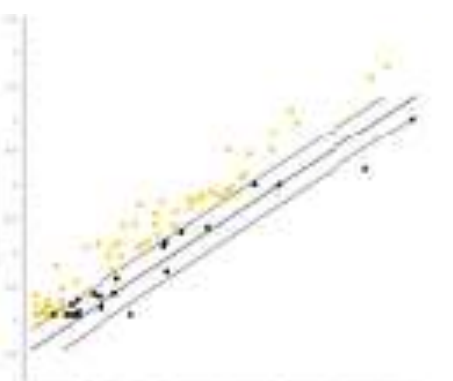

(a)

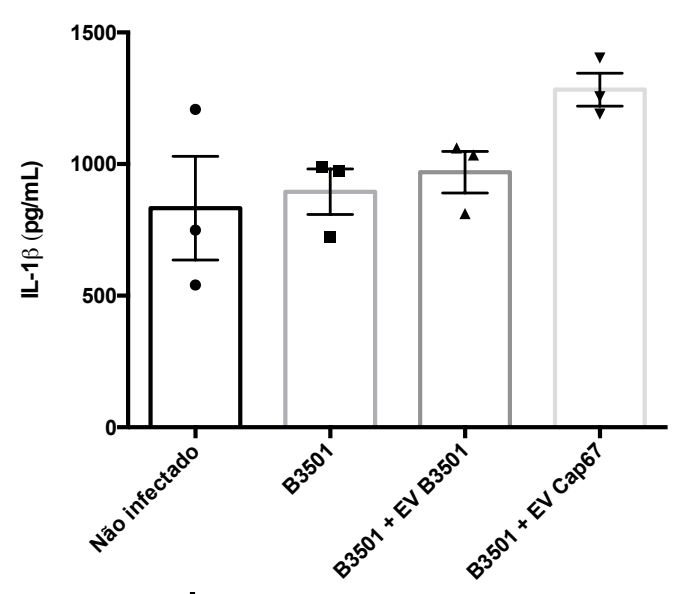

(c)

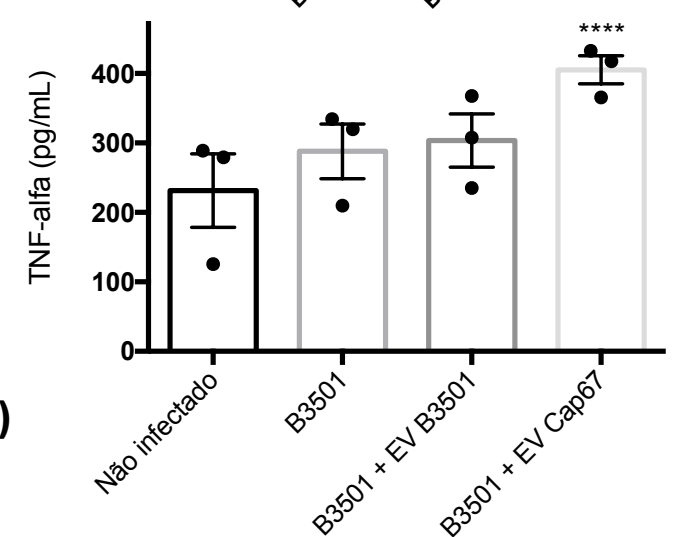

Treated Group Control Group

Treated Group Control Group Cn+ EV Cap $67 \quad$ Cn

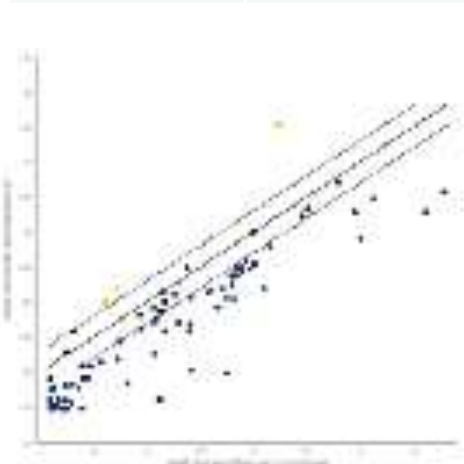

(b)

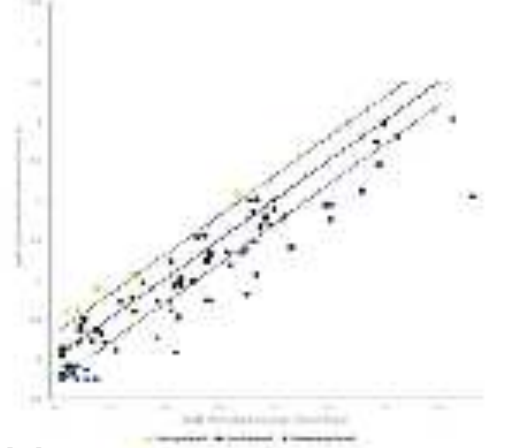

(c)

Figure 5. RT2 PCR array of 84 key genes involved in inflammasome activation done with CDNA obtained from ings of $57 \mathrm{BL} / 6$ mice infected or not with $C$. neoformans B3501 and treated with EVs obtained from B3501 and Cap67 C. neoformans growing on $100 \%$ SDB medium.

\section{Financial support}

Q $C N P q$

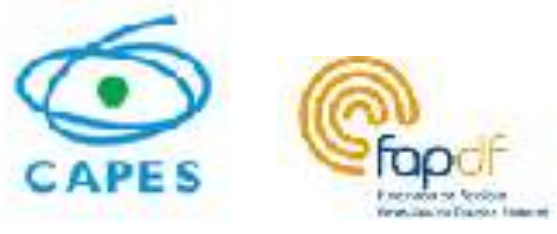

